\title{
Parkinson's Disease Onset Following a Post-traumatic Intracerebral Tension Pneumatocele
}

\author{
Jong Won Kim, Ki Seong Eom \\ Department of Neurosurgery, Wonkwang University School of Medicine, Iksan, Korea
}

The entrapment of intracranial air from the check valve system results in a tension pneumatocele. Tension pneumatoceles should be distinguished from simple pneumatoceles because they are intracranial space-occupying masses that can threaten life. Head trauma is one of the causes in etiopathogenesis of Parkinson's disease. Here, we describe a case of a 50-year-old man who developed Parkinson's disease after a post-traumatic intracerebral tension pneumatocele. In the present case, Parkinson's disease developed 6 months after severe head trauma and 5 months after detection of the tension pneumatocele. To the best of our knowledge, this is the first reported case of Parkinson's disease developed after a post-traumatic delayed tension pneumatocele. Although the exact pathogenic mechanisms underlying the cascade following trauma remain unclear, Parkinson's disease after a tension pneumatocele could be considered a possible complication.

Key Words: Pneumocephalus · Craniocerebral trauma $\cdot$ Parkinson disease $\cdot$ Complications

\section{INTRODUCTION}

A pneumatocele, which is also known as a pneumocephalus or intracerebral aerocele, is defined as the presence of air within any intracranial compartment ${ }^{6}$. The entrapment of intracranial air from the check valve system results in a tension pneumatocele. Tension pneumatoceles should be distinguished from simple pneumatoceles because they are intracranial space-occupying masses that can threaten life ${ }^{6}$. Parkinson's disease is a progressive neurodegenerative disease characterized by impaired motor function as well as nonmotor symptoms ${ }^{2}$. Head trauma is one of the causes in etiopathogenesis of Parkinson's disease. Here, we describe for the first time the case of a 50year-old man who developed Parkinson's disease after a posttraumatic intracerebral tension pneumatocele.

\section{CASE REPORT}

A 50-year-old man was admitted to the emergency room with mental deterioration and stupor after a motor vehicle

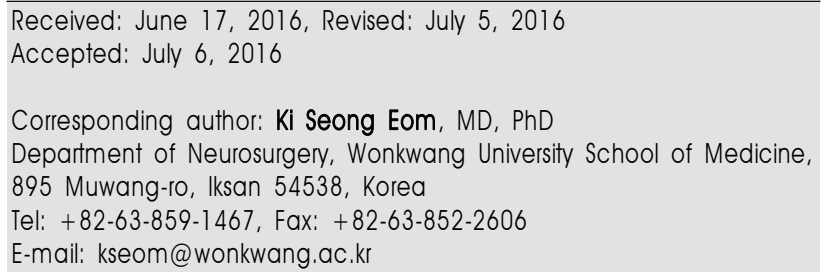

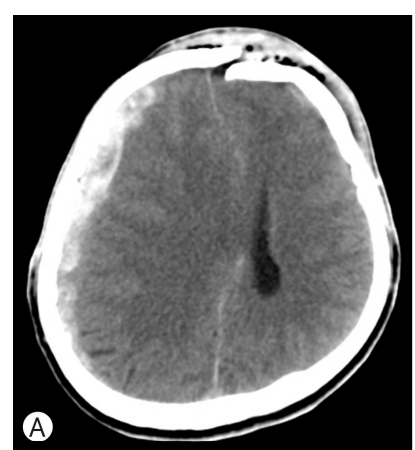
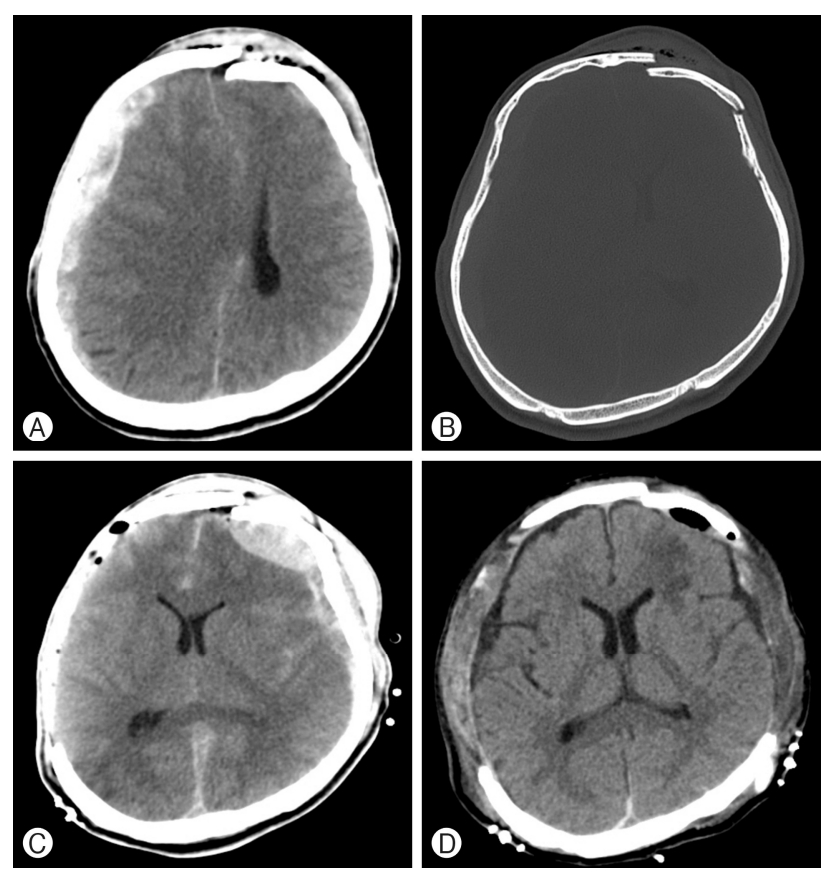

Fig. 1. (A, B) A cranial computed tomography (CT) image showing an acute subdural hematoma (A-SDH) in the right cerebral convexity that caused a midline shift, compression of the ipsilateral ventricle, and a depressed compound comminuted fracture with a small acute epidural hematoma (A-EDH) in the left frontal area. (C) Postoperative CT showing good evacuation of the right A-SDH, a large bony defect along the right cerebral convexity, a larger left A-EDH, and a new A-SDH. (D) Postoperative day-3 CT showing a large bilateral craniectomy and small extraparenchymal pneumocephalus in the left frontal area. 
traffic accident. His consciousness deteriorated to a Glasgow Coma Scale score of 8 (E1, V2, M5), and his left pupil was dilated. Cranial computed tomography (CT) showed an acute subdural hematoma (A-SDH) in the right cerebral convexity that caused a midline shift, compression of the ipsilateral ventricle, and a depressed compound comminuted fracture with a small acute epidural hematoma (A-EDH) in the left frontal area (Fig. $1 \mathrm{~A}, \mathrm{~B})$. He underwent an emergency decompressive craniectomy of the frontotemporoparietal (FTP) bone and evacuation of the A-SDH. Postoperative CT showed good evacuation of the right A-SDH, a large bony defect along the right cerebral convexity, larger left A-EDH, and new A-SDH (Fig. 1C). We performed a left FTP craniectomy and evacuation of the A-SDH and A-EDH. CT performed on postoperative day 3 showed a large bilateral craniectomy and small extraparenchymal pneumocephalus in the left frontal area (Fig. 1D). On postoperative day 7 , his condition and level of consciousness gradually improved, and he became alert. Three weeks later, he presented with a persistent headache without cerebrospinal rhinorrhea. A cranial CT showed a round pneumatocele in the left frontal region (Fig. 2A). A three-dimensional-reconstructed CT showed a depressed bony fracture of the left frontal bone and multiple left facial bone fractures of the sphenoid bone, maxilla, orbital roof, zygomatic arch, and pterygoid bone (Fig. 2B). One week later, follow-up magnetic resonance images of the brain showed an increase in size of the tension pneumatocele in the left frontal base with mass effect (Fig. 2C, D). We removed the tension pneumatocele and repaired the dural defects through a subfrontal approach and reduction of the depressed left frontal bone. Postoperative cranial CT showed very small scattered pneumatoceles and reconstruction of the left frontal bone (Fig. 3A). After removal of the pneumatocele, the patient's headache resolved. Four months later, he underwent a bilateral cranioplasty with autologous bone. Postoperative cranial CT showed no significant abnormal lesions (Fig. 3B). The patient's clinical condition remained satisfactory until 1 month after the bilateral cranioplasty when he presented with slowed movement, impaired posture, and a lack of facial expression. As his typical clinical symptom, he was diagnosed with Parkinson's disease without imaging study including positron emitting tomography and administered Madopar ${ }^{\circledR}$. He was diagnosed with Parkinson's disease and administered Madopar ${ }^{\circledR}$. After medication, his condition showed significant improvement, and we confirmed the diagnosis of Parkinson's disease by his symptom improved. The patient has been doing well 1 year postoperatively.

\section{DISCUSSION}

Various etiologies of pneumatoceles include postoperative,
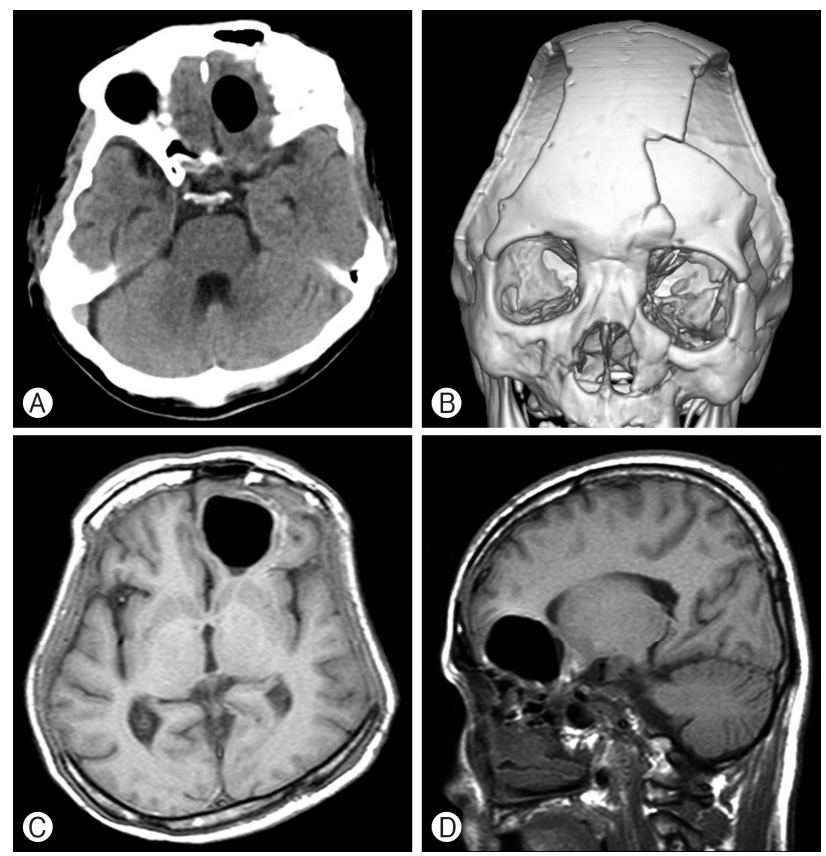

Fig. 2. (A) A cranial computed tomography (CT) image obtained 1 month after bilateral craniectomies showing a round pneumatocele in the left frontal region. (B) A three-dimensional CT recon- struction shows a depressed bony fracture of the left frontal bone and multiple left facial bone fractures. (C, D) One week later, follow-up magnetic resonance images of the brain show a larger tension pneumatocele in the left frontal base with mass effect.
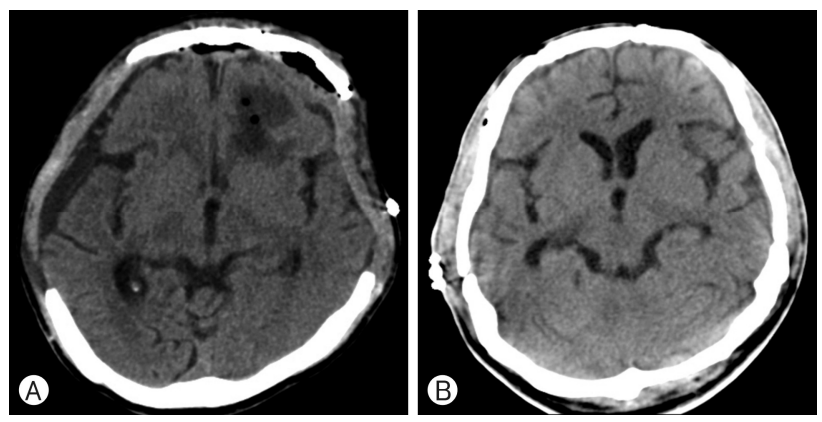

Fig. 3. (A) A cranial computed tomography (CT) image obtained after an operation on a tension pneumatocele and reduction of the depressed left frontal bone showing very small scattered pneumatoceles and reconstruction of the left frontal bone. (B) A cranial CT obtained after bilateral cranioplasties showing no significant abnormal lesions.

post-traumatic, and congenital skull or tegmen tympani defects; tumor erosion through the skull or skull base; infection by gasproducing organisms; postinvasive procedures (lumbar puncture, vetriculostomy); and barotrauma ${ }^{6}$. Two theories have been proposed to explain this phenomenon. The first theory is the ball-valve mechanism, which suggests that positive pressure 
events, such as sneezing, coughing, and Valsalva maneuvers, force air through a cranial defect, which then resists the spontaneous resolution of the air ${ }^{1,6}$. Significant resistance to the outflow of air leads to a tension pneumatocele. The second theory is the inverted-bottle mechanism, which suggests that the negative intracranial pressure gradients that result from drainage of cerebrospinal fluid induce the influx of air ${ }^{6}$. The volume of air is independent of the size of the cranial defect; however, smaller defects are more easily sealed by blood clots or granulation; thus, allowing for gradual reabsorption and spontaneous resolution of the pneumatocele. Most pneumatoceles are small, benign, and cured through non-operative treatments. A significant percentage of pneumatoceles behave like life-threatening space-occupying lesions. Early and precise detection, timely treatment, and appropriate remedy of the source of air are essential for a more favorable clinical outcome. Physicians should confirm the gradual reduction in the amount of intracranial air with serial imaging. A cranial CT scan has proven to be a rapid and dependable technique for observing the accumulation of intracranial air").

Although the etiopathogenesis of Parkinson's disease remains unclear, it is prone to multifactorial and aging-related processes, environmental influences, and genetic predisposition ${ }^{5}$. One factor that has been implicated in the etiopathogenesis of Parkinson's disease is head trauma. Many reports have indicated that head trauma results in Parkinson's disease ${ }^{2,4)}$. Jafari et al. ${ }^{4)}$ performed a meta-analysis to investigate the association between head trauma and the risk for developing Parkinson's disease. Their meta-analysis indicated that a history of head trauma resulting in concussion is associated with a higher risk for developing Parkinson's disease. They insisted that head trauma severe enough to cause a concussion should be considered one of the environmental risk factors for developing Parkinson's disease. Fang et al. ${ }^{2}$ also reported that previous hospitalization due to a head injury was associated with an increased risk of Parkinson's disease; this association could be largely explained by head injuries experienced recently, especially within the year before diagnosis of Parkinson's disease. There are several possible mechanisms for the relationship between head injury and the increased risk of developing Parkinson's disease. The most logical mechanism is neuroinflammation that results from the head injury. Neuroinflammation has been implicated in the development of Parkinson's disease $^{5,7}$. Disruption of the blood-brain barrier due to head trauma can induce leukocyte infiltration and microglial activation ${ }^{7}$. Head trauma may also induce glutamate excitotoxicity and disruption of mitochondrial function, both of which have been implicated in neurodegenerative diseases, including Parkinson's disease $^{3)}$.

In the present case, Parkinson's disease developed 6 months after severe head trauma and 5 months after detection of the tension pneumatocele. It is very difficult to draw conclusions about the relationship between the tension pneumatocele and Parkinson's disease. Undoubtedly, the severe head trauma influenced the development of Parkinson's disease. However, we cannot rule out the possibility that the tension pneumatocele induced the Parkinson's disease because tension pneumatoceles injure the brain parenchyma like other mass-like lesions. Although the specific causes of Parkinson's are unknown, the symptoms are related to a chemical imbalance in the brain caused by brain-cell death.

\section{CONCLUSION}

To the best of our knowledge, this is the first reported case of Parkinson's disease developed after a post-traumatic delayed tension pneumatocele. Development of Parkinson's disease of this patient might be induced severe head trauma and tension pneumatocele gradually. Although the exact pathogenic mechanisms underlying the cascade following trauma remain unclear, Parkinson's disease after a tension pneumatocele could be considered a possible complication. Further research is warranted to clarify this rare complication.

\section{REFERENCES}

1. DelGaudio JM, Ingley AP: Treatment of pneumocephalus after endoscopic sinus and microscopic skull base surgery. Am J Otolaryngol 31:226-230, 2010

2. Fang F, Chen H, Feldman AL, Kamel F, Ye W, Wirdefeldt K: Head injury and Parkinson's disease: a population-based study. Mov Disord 27:1632-1635, 2012

3. Frantseva M, Perez Velazquez JL, Tonkikh A, Adamchik Y, Carlen PL: Neurotrauma/neurodegeneration and mitochondrial dysfunction. Prog Brain Res 137:171-176, 2002

4. Jafari S, Etminan M, Aminzadeh F, Samii A: Head injury and risk of Parkinson disease: a systematic review and meta-analysis. Mov Disord 28:1222-1229, 2013

5. Lees AJ, Hardy J, Revesz T: Parkinson's disease. Lancet 373:20552066, 2009

6. Schirmer CM, Heilman CB, Bhardwaj A: Pneumocephalus: case illustrations and review. Neurocrit Care 13:152-158, 2010

7. Stoll G, Jander S, Schroeter M: Detrimental and beneficial effects of injury-induced inflammation and cytokine expression in the nervous system. Adv Exp Med Biol 513:87-113, 2002 